\title{
Healthcare-Associated Infections: New Initiatives and Continuing Challenges
}

Michael Gardam, Paige Reason and Leah Gitterman

\begin{abstract}
Healthcare-associated infections are a major cause of patient morbidity and mortality. Fortunately for patients and the healthcare system, there is increasing interest in this field and the growing realization that many of these infections are highly preventable. We explore some of the newer and more promising strategies for decreasing infections, including the use of practice bundles, behavioural change strategies, hand hygiene auditing, public reporting of infection rates and antimicrobial stewardship. We also identify several areas where improvement is needed, including empowering patients to prevent infections, building safer healthcare facilities and accepting the limitations of the evidence supporting some infection control interventions.
\end{abstract}

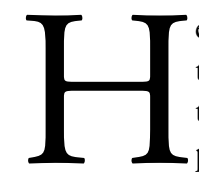

ealthcare-associated infections (HAIs) are infections that occur in patients as a result of their being treated for another, unrelated issue. For example, a patient admitted to hospital to receive a life-saving cardiac procedure may initially do well post-procedure, only to later succumb to an infection that developed as a result of an intervention such as a the use of central intravenous line or ventilation. A patient may also develop a life-threatening infection such as Clostridium difficile colitis by first picking up the organism through contaminated healthcare worker hands or contaminated equipment, and then receiving antibiotics that wipe out the patient's normal intestinal flora and allow $C$. difficile to thrive.
It has been estimated that well over 200,000 Canadians develop such infections each year, resulting in more than 8,000 deaths (Office of the Auditor General of Ontario 2008). While this number is shocking, it is perhaps telling that patient safety organizations only relatively recently began focusing on HAIs as preventable patient safety issues. While attempts to control infections date back to Florence Nightingale during the Crimean War, the antibiotic era resulted in an attitude shift in which treating infections received more focus than preventing them. For many decades, HAIs have been seen as "a cost of doing

\section{Many of our traditional approaches do not work well in settings where "culture eats strategy for breakfast."}

business" (Gardam et al. 2009). Fortunately for patients and our healthcare system as a whole, attitudes are slowly changing as more and more evidence points to different interventions that can profoundly decrease infection rates.

This renewed interest in HAIs has resulted in a number of promising new approaches. It has also, however, shone a light on the sobering fact that some traditional infection prevention and control approaches do not work very well. In this article, we highlight some of the promising new approaches as well as challenging areas where we believe the infection prevention and control community needs to rethink its approaches. 


\section{New Approaches Practice Bundles}

Practice bundles are groups of clinical or other practices that have been studied and found to have a positive impact on infection rates (Institute for Healthcare Improvement 2011). Research and real-world experience over the past 10 years has clearly shown that, for some types of HAIs, using the practice bundle approach can often have a dramatic impact (Jain et al. 2011; Resar et al. 2005). Typically, the individual practice changes are remarkably simple. For example, central intravenous line infections can be significantly reduced in part by choosing appropriate insertion sites; treating the line insertion as a true sterile procedure, that is, requiring sterile drapes and using chlorhexidine alcohol skin preparation; and removing the line when it is no longer needed (Safer Healthcare Now! 2009a). Similarly, surgical site infections and ventilator-associated pneumonias can also be reduced through clear-cut interventions (Safer Healthcare Now! 2009b, 2010). Often, the spread of bundled approaches has been facilitated by collaboratives, where teams from different organizations work together while implementing practice bundles to learn from each other, share practices and provide mutual support (Institute for Healthcare Improvement 2003).

However, practice bundles cannot be the only answer or approach to preventing HAIs. A central, necessary condition of the practice bundle approach is that the event or intervention can be isolated in time, controlled, standardized and audited to ensure compliance. While this approach makes sense for perhaps most causes of HAIs that fit this condition, it is less obvious how it might be applied to circumstances that are far less controlled, such as healthcare worker compliance with hand hygiene.

\section{Adopting a Complexity Science Approach to Complex Infection Control Challenges}

Hand in hand with the growing recognition of the power of practice bundles in bringing about improvements was the recognition that this approach is not applicable to all infection control challenges. Proven infection prevention practices have been known for years, yet most healthcare organizations have been unable to achieve consistently high rates of adherence to these practices. Traditional strategies typically involve the reinforcement of healthcare worker hand hygiene, environmental cleaning, surveillance for colonized or infected patients and varying forms of isolation when certain infections are detected. Some components of the control measures to prevent the spread of hospital "superbugs" between patients may be amenable to a practice bundle approach (i.e., standardizing how to clean a patient room), but many of the factors that influence spread are deeply rooted in behaviour and inherently non-linear (Gardam et al. 2009; Lindberg et al. 2008). Experience over the past several decades and the study of complex adaptive systems have identified that many of our traditional approaches to more simple problems, such as checklists and the implementation of "best practices," do not work well in settings where "culture eats strategy for breakfast" (Gardam et al. 2010). Rather, approaches for such challenges need to recognize the need for local variation, acknowledge local culture and recognize the requirement for multiple small fixes rather than one big solution to a problem (Glouberman and Zimmerman 2002). Often, those attempting to implement a particular practice change hear from front-line staff, "That isn't the way we do things here." Rather than ignore this, complexity science suggests that this "social immune response" should be acknowledged and that staff be allowed to create an intervention that works in their setting.

Recognizing this different approach and the need for local solutions, engagement and empowerment of those who are deeply connected to the problem at hand often can yield surprising results. Positive deviance is one such front-line empowerment strategy that has been used with success in tackling complex infection control challenges such as hand hygiene (Marra et al. 2010). The Canadian Positive Deviance Project used this approach and other front-line engagement strategies termed liberating structures (Social Invention Group 2007) to help control the spread of superbugs in five acute care hospitals. These complexity science-based methodologies help to guide groups in the midst of a problem toward solutions that are situation appropriate and that work for them in their setting. It was found that the use of these approaches over an 18-month period not only decreased rates of methicillin-resistant Staphylococcus aureus MRSA, vancomycin-resistant enterococci (VRE) and $C$. difficile by an overall average of $56 \%$, but also had a significant impact upon how staff relate and interact with one another (Reason et al. 2012). Simply put, infections are prevented because staff act differently and implement their own solutions. Remarkably, these approaches rarely require significant financial investment.

\section{Public Reporting of Infections}

The measurement and feedback of data are central tenets of any improvement work: how do you know where to go if you don't know where you are? The recent trend in mandatory public reporting of infection rates in several Canadian provinces has taken this to a higher level. Now, not only can a facility benchmark with itself and track its performance, it can also benchmark with other facilities. In our experience, public reporting in Ontario has helped improvement processes by drawing much broader attention to the issues at hand and by enabling facilities at the higher end of the infection spectrum to realize that they might have a bigger problem than they thought.

These positive effects must, however, be balanced by several caveats. Because public reporting data rarely control for known factors that influence infection rates, with the exception of hospital type and size, it is very difficult to meaningfully compare infection rates between facilities; yet the public and the media 
do exactly that. The indicators used may be chosen because they are convenience rather than meaningful. For example, Ontario's reporting on MRSA and VRE bloodstream infections, while simple and largely bias free, is not that helpful for quality improvement simply because these are very rare events in most organizations (Ontario Ministry of Health and Long-Term Care 2011). A consistent rate of zero can obscure the fact that many other more common infections are ongoing and causing harm. Finally, process measures such as hand hygiene can be quite problematic as public accountability measures because current auditing processes can introduce substantial biases into rates, making it appear that hand hygiene compliance is excellent when it may not be (Muller and Detsky 2010).

\section{Hand Hygiene Compliance Programs}

Although hand hygiene was identified roughly 150 years ago as a major means of controlling the spread of infectious agents to patients (Semmelweis 1983), only recently have multiple organizations and countries made improving hand hygiene a priority that requires specific resources, education and interventions. Part of the reason for this long delay may be that, while the act of cleaning one's hands is simple, the behaviour that drives compliance (or non-compliance) is remarkably complex (World Health Organization 2009). It remains common for administrators and leaders to be shocked and disappointed about how difficult it is to make significant progress with compliance.

\section{Likely all of us have heard a physician say, "I'll give you an antibiotic, just in case."}

An abundance of literature suggests that improving hand hygiene requires a multi-faceted approach, combining elements of social marketing, human factors engineering, education, strong leadership support and data feedback (World Health Organization 2009). While a focused educational or awareness campaign often temporarily increases compliance, the increase typically dissipates unless behaviour and expectations fundamentally change.

As mentioned previously, measurement is central to any change process, yet hand hygiene compliance measurement involving human auditors is both costly and subject to substantial bias. Not only does being observed have the potential to temporarily modify behaviour during the auditing period (i.e., the act of measuring influences the results), but because it is costly and labour intensive, only small numbers of measures can be obtained over short periods of time. The knowledge that rates will be reported publically only further inflates this bias (Muller and Detsky 2010).

Emerging technological approaches to hand hygiene auditing involving the use of radio frequency identification and other technologies have the potential to profoundly alter our under- standing of hand hygiene compliance (Edmond et al. 2010; Levchenko et al. 2011). Through small transmitters worn by staff, these technologies can capture hand hygiene compliance at every moment of the day, thus providing potentially thousands of data points per day. Some systems incorporate reminders into the system so that staff are prompted to clean their hands should they miss an opportunity. Provided that these hand hygiene data are reported back to healthcare workers in ways that encourage improvement, these technologies have the potential to help identify problem areas and encourage behavioural change.

\section{Antimicrobial Stewardship}

Simply put, antimicrobial stewardship programs attempt to ensure that patients receive the right antibiotic at the right time for the right duration (Dellit et al. 2007). This is not a new concept; however, there is considerable renewed interest in this field, fuelled by the spread of highly drug-resistant and difficult-to-treat organisms such as carbapenemase-producing gram-negative bacteria (Moellering 2010) and antibiotic-associated diseases such as $C$. difficile diarrhea (Kelly and LaMont 2008). These significant threats to patient safety stem at least partially from the widespread use and misuse of antibiotics that occurs in all healthcare sectors. Unfortunately, the prescribing of antibiotics has been viewed as a benign intervention, and likely all of us at some point have heard a physician say, "I'll give you an antibiotic, just in case." While this behaviour comes from concern about missing a treatable bacterial infection, there needs to be greater recognition that antibiotics can cause harm. In addition, some antibiotics are quite expensive and, yet, are being given for questionable indications.

The current stewardship model relies heavily on intervention by trained pharmacists and specialty physicians; however, most healthcare settings, including hospitals, do not have access to such resources (Johannsson et al. 2011). Stewardship needs to evolve from an intervention that only academic and large community hospitals can afford into a far more flexible program where different aspects of stewardship can be implemented in almost any setting, including long-term care and outpatient clinics.

\section{Continuing Challenges Patient Engagement}

Despite some progress, patients and families remain largely sidelined from the infection control process: in general, rather than patients being engaged in creating and implementing policies, control measures are done to patients by healthcare workers. Patients and their families are seen as potentially mobile sources of contagion, rather than people who may well have insights into possible solutions. Fortunately, there are some examples of settings where these individuals are becoming more involved (Bittle and LaMarche 2009).

Furthermore, there continue to be confusion surrounding 
what carrying a hospital "superbug" means, an inconsistent application of control measures across healthcare settings and vagueness surrounding the risks to patients and others upon discharge. Indeed, infection control measures often stop upon exit from the hospital, even though patients likely access ambulatory care. Patients want to know what the transmission risk is to their family members, how long they will potentially carry the organism etc. More work needs to be done to address these gaps and inconsistencies in practice across the continuum of care. This can start with a simple conversation with patients and their families, answering questions and providing education, and allowing them to be a part of the solution, not just passive recipients of care.

\section{Never-Ending Search for a Common Approach}

Unfortunately, much of infection control practice is not black or white but, rather, many shades of grey. For example, there is some literature indicating that alcohol-based hand rubs (ABHR) do not kill $C$. difficile spores, leading some to argue that one should only clean one's hands with soap and water when caring for patients with this infection (Jabbar et al. 2010; Oughton et al. 2009). Others argue that while it is true that ABHRs do not kill $C$. difficile spores, insisting upon soap and water washing will dramatically decrease hand hygiene compliance since it takes longer and is more drying to the hands, and often sinks are not readily accessible. Both of these are valid points of view and, not surprisingly, various expert bodies have released differing recommendations (Ontario Ministry of Health and Long-Term Care 2010; Siegel et al. 2007). Similarly, rings have been shown to harbour potentially pathogenic organisms, even after one performs hand hygiene (World Health Organization 2009). Requiring rings to be removed prior to handwashing would solve this problem but, again, would likely decrease compliance with hand hygiene.

Searching for the "correct" answer when there isn't one can lead to a stalemate and hamper progress. Rather, we suggest that institutions follow the approaches make sense in their context. Perhaps, over time, enough evidence will be gathered to clearly delineate a preferred approach.

\section{Healthcare Design}

Despite considerable, robust evidence that healthcare design has a large role to play in preventing the spread of communicable agents, Canada has been slow to adopt single-patient room design standards that have been accepted in other countries such as the United States (American Institute of Architects 2006). It has been shown that having roommates is a very potent risk factor for acquiring a communicable organism (Hamel et al. 2010). Furthermore, single-patient rooms have been shown to have multiple other patient safety and comfort benefits that ultimately result in decreased lengths of stay and better outcomes (Ulrich et al. 2008). All told, it is estimated that the upfront capital costs are rapidly recouped over the lifetime of the facility (Ulrich et al. 2008). Fortunately, the Canadian Standards Association has recently released new guidelines that support the use of singlepatient rooms (Canadian Standards Association 2011).

\section{Healthcare Culture}

Perhaps the most central factor that continues to drive the spread of infectious agents in hospital is healthcare worker culture. As mentioned previously, until recently HAIs were not seen as patient adverse events. Even today, there is far more innate comprehension by both healthcare workers and patients themselves that a critical medication error (e.g., giving the wrong dose of chemotherapy) is tragic and preventable, than there is that a fatal case of $C$. difficile diarrhea resulting from poor environmental cleaning and antibiotic misuse is tragic and preventable (Gardam et al. 2009).

\section{Searching for the "correct" answer when there isn't one can lead to a stalemate.}

We have stated before that HAIs have perhaps more in common with social problems such as littering than with our traditional perception of an adverse event, which tends to have a more linear association between cause and effect. During a typical week-long hospital stay, patients are literally exposed to thousands of diverse human and environmental contacts that may lead to them acquiring a hospital superbug or developing a device-related infection - the hospital is a truly complex environment. While there may only be one exposure that ultimately leads to infection, it is nearly impossible to determine which one was responsible.

Thus, all pieces of the healthcare system have the potential to harm patients and, importantly, the power to protect them. Fortunately for our patients, once we acknowledge the central role of culture in patient safety, we can begin to successfully address problems by using approaches that work with culture rather than against it.

\section{Conclusion}

The field of infection prevention and control is maturing, thanks to renewed interest by the healthcare community and patients and the realization that effective, low-cost strategies to prevent infections exist. New approaches to data measurement and feedback are emerging that may help shine a light on current problems that contribute to an unsafe environment.

While there will always be a necessary role for commandand-control approaches during dangerous outbreaks, it is our hope that this field will continue toward a model where all those involved in the problem will be recognized as part of the 
solution. We have witnessed the ability of front-line empowerment to enable significant positive change on multiple occasions throughout Canada. Every organization has individuals who are fed up with the status quo and who have ideas on how to make things better, yet our traditional hierarchy does not allow them to have a voice. By traditional leadership allowing these individuals to lead change, encouraging others to try their ideas, and learning to step back and move toward a facilitation and support role, it is possible to achieve what we were unable to accomplish with our usual approaches. $\mathrm{HQ}$

\section{References}

American Institute of Architects. 2006. Guidelines for Design and Construction of Health Care Facilities. Washington, DC: Facility Guidelines Institute.

Bittle, M.J. and S. LaMarche. 2009. "Engaging the Patient as Observer to Promote Hand Hygiene Compliance in Ambulatory Care." Joint Commission Journal on Quality and Patient Safety 35(10): 519-25.

Canadian Standards Association. 2011. Health Care Facilities Standard. Mississauga, ON: Author. Retrieved December 2, 2011. <http://www. $\mathrm{csa} . \mathrm{ca} / \mathrm{cm} / \mathrm{ca} / \mathrm{en} /$ news/article/csa-writes-prescription-for-design-andconstruction-of-hospitals-and-health-care-facilities $>$.

Dellit, T.H., R.C. Owens, J.E. McGowan, D.N. Gerding, R.A. Weinstein, J.P. Burke et al. 2007. "Infectious Diseases Society of America and the Society for Healthcare Epidemiology of America: Guidelines for Developing an Institutional Program to Enhance Antimicrobial Stewardship." Clinical Infectious Diseases 44: 159-77.

Edmond, M.B., A. Goodell, W. Zuelzer, K. Sanogo, K. Elam and G. Bearman. 2010. "Successful Use of Alcohol Sensor Technology to Monitor and Report Hand Hygiene Compliance." Journal of Hospital Infection 76: 364-65.

Gardam, M.A., C. Lemieux, P. Reason, M. van Dijk and V. Goel. 2009. "Healthcare Associated Infections as Patient Safety Indicators." Healthcare Papers 9(3): 8-24.

Gardam, M.A., P. Reason and L. Rykert. 2010. "Healthcare Culture and the Challenge of Preventing Healthcare-Associated Infections." Healthcare Quarterly13: 116-20.

Glouberman, S. and B. Zimmerman. 2002. Complicated and Complex Systems: What Would Successful Reform of Medicare Look Like? Washington, DC: Plexus Institute. Retrieved October 11, 2011. $<$ www.plexusinstitute.org $>$.

Hamel, M., D. Zoutman and C. O'Callaghan. 2010. "Exposure to Hospital Roommates as a Risk Factor for Health Care-Associated Infection." American Journal of Infection Control 38: 173-81.

Institute for Healthcare Improvement. 2003. The Breakthrough Series: IHI's Collaborative Model for Achieving Breakthrough Improvement. Cambridge, MA: Author. Retrieved October 11, 2011. <www.ihi.org>.

Institute for Healthcare Improvement. 2011. What Is a Bundle? Cambridge, MA: Author. Retrieved October 11, 2011. <http://www. ihi.org/knowledge/Pages/ImprovementStories/WhatIsaBundle.aspx>.

Jabbar, U., J. Leischner, D. Kasper, R. Gerber, S. Sambol, J. Parada et al. 2010. "Effectiveness of Alcohol-Based Hand Rubs for Removal of Clostridium difficile Spores from Hands." Infection Control and Hospital Epidemiology 31(6): 565-70.

Jain, R., S. Kralovic, M. Evans, M. Ambrose, L. Simbartl, S. Obrosky et al. 2011. "Veterans Affairs Initiative to Prevent Methicillin-Resistant
Staphylococcus aureus Infections." New England Journal of Medicine 364: 1419-30.

Johannsson, B., S. Beekmann, A. Srinivasan, A. Hersh, R. Laxminarayan and P. Polgreen, on behalf of the Infectious Diseases Society of America Emerging Infections Network. 2011. "Improving Antimicrobial Stewardship: The Evolution of Programmatic Strategies and Barriers." Infection Control and Hospital Epidemiology 32(4): 367-74.

Kelly, C. and J. LaMont. 2008. "Clostridium difficile - More Difficult Than Ever.” New England Journal of Medicine 359: 1932-40.

Levchenko, A.I., V.M. Boscart and G.R. Fernie. 2011. "The Feasibility of an Automated Monitoring System to Improve Nurses' Hand Hygiene." International Journal of Medical Informatics 80(8): 596-603. [Epub 2011 May 20].

Lindberg, C., J. Lloyd and P. Buscell. 2008. RWJF Final Report. Washington, DC: Plexus Institute. Retrieved December 2, 2011. <http://www.plexusinstitute.org/resource/collection/6528ED299907-4BC7-8D00-8DC907679FED/55726FNR_-_RWJF_Final_ Report_-_PD_MRSA.pdf>.

Marra, A., L. Guastelli, C. de Araujo, J. Santos, L. Lamblet, M. Silva et al. 2010. "Positive Deviance: A New Strategy for Improving Hand Hygiene Compliance." Infection Control and Hospital Epidemiology 31(1): 12-20.

Moellering, R. 2010. "NDM-1 - A Cause for Worldwide Concern." New England Journal of Medicine 363: 2377-79.

Muller, M. and A Detsky. 2010. "Public Reporting of Hospital Hand Hygiene Compliance - Helpful or Harmful?” Journal of the American Medical Association 304(10): 1116-17.

Office of the Auditor General. 2008. Special Report: Prevention and Control of Hospital-Acquired Infections. Toronto, ON: Author. Retrieved October 11, 2011. <http://www.auditor.on.ca/en/reports_ en/hai_en.pdf>.

Ontario Ministry of Health and Long Term Care, Provincial Infectious Diseases Advisory Committee. 2010. Annex C. Testing, Surveillance and Management of Clostridium difficile in all Healthcare Settings. Toronto, ON: Author. Retrieved October 12, 2011. <http://www.oahpp.ca/ resources/pidac-knowledge/best-practice-manuals/testing-surveillanceand-management-of-clostridium-difficile.html>.

Ontario Ministry of Health and Long Term Care. 2011. Vancomycin Resistant Enterococci. Toronto, ON: Author. Retrieved October 12, 2011. <http://www.health.gov.on.ca/patient_safety/pro/vre/vre_pro.html>.

Oughton, M., V. Loo, N. Dendukuri, S. Fenn and M. Libman. 2009. "Hand Hygiene with Soap and Water Is Superior to Alcohol Rub and Antiseptic Wipes for Removal of Clostridium difficile." Infection Control and Hospital Epidemiology 30(10): 939-44.

Reason, P., L. Rykert, B. Zimmerman, L. Gitterman, J. Christian, N. Crowcroft et al. 2012. "Decreasing Healthcare-Associated Infections through Enabling the Front-Line.” Manuscript in Preparation.

Resar, R., P. Pronovost, C. Haraden, T. Simmonds, T. Rainey and T. Noland. 2005. "Using a Bundle Approach to Improve Ventilator Care Processes and Reduce Ventilator-Associated Pneumonia." Joint Commission Journal on Quality and Patient Safety 31 (5): 243-48.

Safer Healthcare Now! 2009a. Getting Started Kit: Prevent Central Line Infections How to Guide. Edmonton, AB: Author. Retrieved October 11, 2011. <www.saferhealthcarenow.ca>.

Safer Healthcare Now! 2009b. Getting Started Kit: Prevention of Ventilator-Associated Pneumonia in Adults and Children How to Guide. Edmonton, AB: Author. Retrieved October 11, 2011. <www.saferhealthcarenow.ca>. 
Safer Healthcare Now! 2010. Prevent Surgical Site Infections: Getting Started Kit. Edmonton, AB: Author. Retrieved October 11, 2011. $<$ www.saferhealthcarenow.ca>.

Semmelweis, I. 1983. The Etiology, Concept and Prophylaxis of Childbed Fever (K.C. Carter, Trans.). Madison, WI: The University of Wisconsin Press.

Siegel, J.D., E. Rhinehart, M. Jackson, L. Chiarello and the Healthcare Infection Control Practices Advisory Committee. 2007. Guideline for Isolation Precautions: Preventing Transmission of Infectious Agents in Healthcare Settings. Atlanta, GA: Centers for Disease Control and Prevention. Retrieved October 12, 2011. <http://www.cdc.gov/ ncidod/dhqp/pdf/isolation2007.pdf>.

Social Invention Group. 2007. Liberating Structures. Seattle, WA: Author. Retrieved October 12, 2011. <http://socialinvention.net/ LiberatingStructuresHome.aspx $>$.

Ulrich, R.S., C.M. Zimring, X. Zhu, J. Dubose, H.B. Seo and Y.S. Choi et al. 2008. A Review of the Research Literature on Evidence Based Healthcare Design, White Paper Series 5/5, Evidence-Based Design Resources for Healthcare Executives. Concord, CA: The Center for Health Design.
World Health Organization. 2009. WHO Guidelines on Hand Hygiene in Healthcare. Geneva, Switzerland: Author. Retrieved October 11, 2011. <http://whqlibdoc.who.int/publications/2009/9789241597906_eng. pdfs.

\section{About the Authors}

Michael Gardam, MSc, MD, CM, MSc, FRCPC, is a member of the Infection Prevention and Control Unit, University Health Network (UHN), in Toronto, Ontario; the STOP Infections Now collaborative, Safer Healthcare Now!; and the Division of Infectious Diseases, Faculty of Medicine, at the University of Toronto. He can be contacted at 416-340-3758 or by e-mail at michael.gardam@uhn.on.ca.

Paige Reason, MPH, is a member of the Infection Prevention and Control Unit, UHN; and the STOP Infections Now collaborative, Safer Healthcare Now!

Leah Gitterman, MHSc, is a member of the Infection Prevention and Control Unit, UHN, and the STOP Infections Now collaborative, Safer Healthcare Now!

\section{Make quality your priority. Choose Accreditation Canada.}

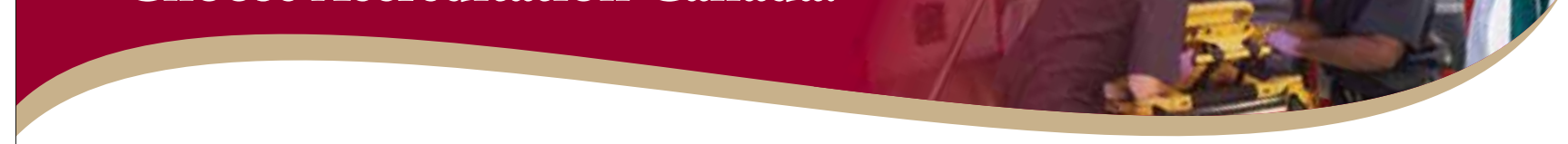

Accreditation Canada provides health organizations with a comprehensive evaluation process, including an external peer review, to assess the quality of their services based on national standards of excellence.

Our Emergency Medical Services (EMS) Standards focus on response, transport, and care, and now include enhanced content on EMS communication centres.

The EMS Standards will help your organization

$\checkmark$ Incorporate quality into everyday service

$\checkmark$ Bring safety to the forefront

$\checkmark$ Manage and prevent infection

$\checkmark$ Focus on clients' needs

$\checkmark$ Enhance credibility

To learn more

E-mail LearnMore@accreditation.ca

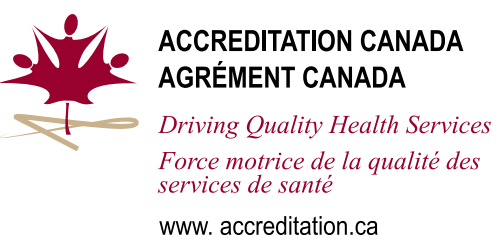

Accreditation Canada is accredited by the International Society for Quality in Health Care 